\title{
myAIM - mein Ziel: Allgemeine Innere Medizin
}

\section{Sven Streit}

Dr. med., Projektleiter myAIM, Research Fellow am Berner Institut für Hausarztmedizin BIHAM

Die Allgemeine Innere Medizin (AIM), ob im Spital oder in der Praxis, ist bei Studenten und Assistenzärzten der beliebteste Facharzttitel. 2011 entstand ein gemeinsames Weiterbildungsprogramm, 2013 die gemeinsame Facharztprüfung und nun gibt es eine gemeinsame Fortbildungsverordnung. Zeit, sich auch für den Nachwuchs einzusetzen: Auf der neuen gemeinsamen Website «myAIM» sind alle wichtigen Informationen für Assistenzärztinnen und -ärzte zusammengestellt.

Wir Hausärzte und Generalisten im Spital haben vieles gemeinsam. Zum Bespiel gemeinsame Ziele: Wir wollen das Beste für unsere Patienten, wir möchten eine sinnvolle Medizin und einen attraktiven Beruf.

Die neue Website myAIM soll die Visitenkarte für die neue Fachgesellschaft SGAIM und ihren Berufsstand sein.

Wer heute aber Antworten auf seine Fragen zur Weiterbildung AIM Antworten sucht, verzweifelt angesichts der Fülle von Informationen auf Internetseiten, bei Informationsstellen und in Newslettern. Wer überblickt noch diese Flut von Informationen? Wer

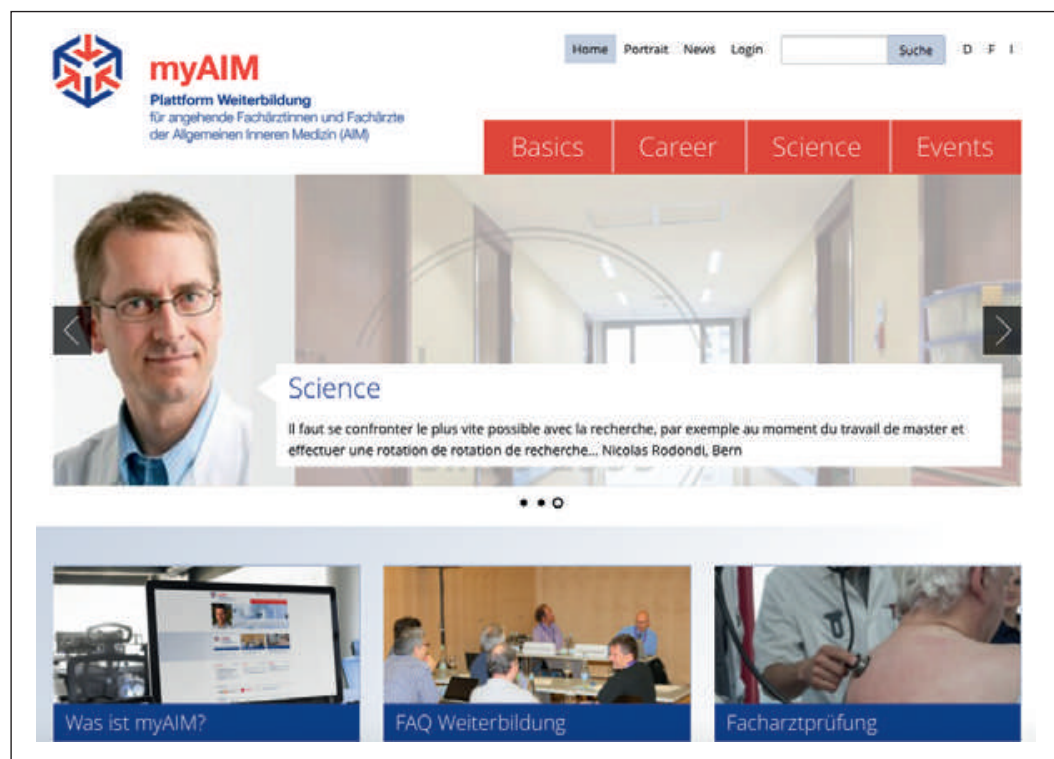

Willkommen auf myAIM: die Welt der Weiterbildung AIM im Überblick auf der Hauptseite. kennt nicht die Gedanken «Hätte ich das nur früher gewusst», "Wo finde ich das bloss?" "An wen soll ich mich wenden?» "Was ist überhaupt ...»? Diese bei Assistenzärzten häufigen Fragen zeigen, dass zu viele Informationen im Netz zu weit verstreut sind. Dies einerseits, weil immer mehr Organisationen ihren Webauftritt ausbauen und andererseits, weil immer mehr neue Organisationen und Netzwerke entstehen. Beides geschieht in der guten Absicht, sich für die Allgemeine Innere Medizin - ob im Spital oder in der Hausarztmedizin - stark zu machen. Für den Nachwuchs bleibt es aber schwierig, den AIM-Wissensberg zu erklimmen.

\section{Übersichtlich - verständlich - aktuell}

Ärzte mögen es, wenn sie sich zum Einlesen in ein Thema auf eine glaubwürdige, kurzgefasste und gut verlinkte Seite wie z.B. Uptodate ${ }^{\circledR}$ verlassen können. Mehrmals wurde versucht, ein solches Konzept auch für Assistenzärzte in Weiterbildung umzusetzen, damit sie "up to date» bleiben können. Auf Anregung des Berner Instituts für Hausarztmedizin (BIHAM) gelang es schliesslich mit Hilfe der JHaS (Junge Hausärzte und -ärztinnen Schweiz), genügend Ärzteorganisationen zu bewegen, ihre Synergien hierfür zu nutzen. Ziel war eine gute Webseite - für gut informierten Nachwuchs - und als gute Möglichkeit, Kollegen zu erreichen (z.B. für interessante Veranstaltungen). Wir hoffen hier auf eine «Win-win-win-Situation». Die neue Website myAIM sollte die Visitenkarte für die neue Fachgesellschaft SGAIM (Schweizersiche Gesellschaft für Allgemeine Innere Medizin; aus SGAM und SGIM) und ihren Berufsstand werden. 


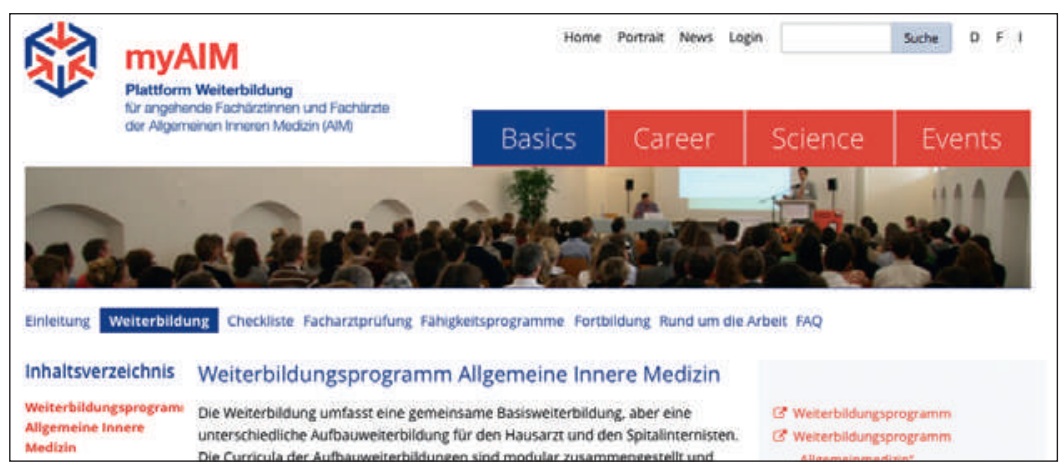

Die Unterseite «Basics»: Erklärt wird alles rund um das Weiterbildungsprogramm.

JHaS und Swiss Young Internists (SYI) stellten eine 6-köpfige Redaktion zusammen, um die Inhalte für myAIM zu bestimmen. Die Redaktoren kommen alle aus dem weiten Feld der Allgemeinen Inneren Medizin und sind Assistenz-, Ober- oder Hausärzte. Die beiden Fachgesellschaften SGAM und SGIM waren bereit, als Trägerorganisationen zu fungieren, Sven Streit wurde die Projektleitung für myAIM übertragen. Dank grosszügiger Subventionierung durch das Bundesamt für Gesundheit BAG konnte das Projekt 2013 begonnen werden.

myAIM will vor allem eines: Überblick schaffen. Dazu wurde die Webseite in vier Kapitel unterteilt und auf gut verständliche Sprache geachtet. Es finden sich viele Links auf Originalseiten. So werden Doppelspurigkeiten vermieden und die Aktualität der Informationen garantiert. myAIM wird kein "Telefonbuch", das nach einem Jahr wieder veraltet ist, sondern hält Schritt mit den laufenden Veränderungen rund um die AIM.

\section{Basics - alles zum Thema Weiterbildung}

myAIM erklärt, wann ein e-Logbuch auszufüllen ist, wie viele arbeitsplatzbasierte Assessments jährlich durchzuführen sind und gibt Tipps, wie Beruf und Familie vereinbart werden können. Eine Checkliste aus dem Weiterbildungsprogramm erklärt, wie der Facharzt AIM zu erlangen ist.

\section{Career - Was nach dem Facharzt kommt: Profil Hausarzt und Spitalarzt}

Für junge Ärzte und Ärztinnen in Weiterbildung ist es wichtig, Einblick in verschiedene Karrieremöglichkeiten zu erhalten. Wichtig erscheint uns hierbei, auch Möglichkeiten wie Mentoring, Fähigkeitsausweise, Laborkurse, Sonographie usw. vorzustellen. Nur so können sie erkennen, was neben dem Facharzttitel für ihre spätere Tätigkeit wichtig sein wird. Abgerundet wird diese Rubrik mit Testimonials - Kollegen aus Praxis und Spital geben Einsicht in ihre Arbeit.

\section{Science - Forschung AIM bekannt machen}

Science erklärt, warum Forschung im Gebiet AIM wichtig ist und porträtiert ausgezeichnete Schweizer Forscher als positive Beispiele. An einem «Schwarzen Brett" können Dissertationen oder Kooperationen ausgeschrieben werden. Herausragende Publikationen werden laufend gesammelt.

\section{Events - von Jungen für Junge}

Mit der dynamischen Datenbank können Veranstaltungen für Assistenzärzte gesucht werden, geordnet nach Regionen oder Interesse. Nach Registrierung kann man diesen Service als regelmässige E-MailBenachrichtigung abonnieren

\section{myAIM - ganz individuell}

Neben Übersicht, Verständlichkeit und Aktualität macht die Attraktivität von myAIM aber vor allem eines aus: individualisierte Informationen. So können sich Benutzer registrieren lassen und angeben, welche Informationen zu welcher Zeit über welche Kanäle bei ihnen eintreffen sollen. Wer sich für AIMStellen im Teilzeitbereich interessiert, kann sich Angebote über unseren Partner «Jobmed" von VSAO Mediservice zustellen lassen. Wer interessante Weiter- und Fortbildungen in seiner Region nicht verpassen will, erhält einen Reminder per E-Mail oder Social Media. Die myAIM-Redaktion verfasst regelmässig auf Assistenzärzte zugeschnittene News, die ebenfalls abonniert werden können.

\section{Online ab 20. Mai 2015}

Die Website geht bald online. Dank vieler Partner wird sie aktuell bleiben. Wir arbeiten zusammen mit den Fachgesellschaften für Allgemeinmedizin (SGAM), Allgemeine Innere Medizin (SGIM), dem Schweizerischen Institut für ärztliche Weiter- und Fortbildung (SIWF), der Stiftung zur Förderung der Weiterbildung in Hausarztmedizin (WHM), dem Cursus romand de médecine de famille (CRMF), Hausärzte Schweiz (MFE) und sämtlichen Instituten für Hausarztmedizin. An dieser Stelle sei allen für die Bereitschaft gedankt, sich gemeinsam für den Nachwuchs an Fachärzten in Allgemeiner Innerer Medizin - in der Hausarztmedizin und im Spital - einzusetzen.

Auf YouTube wird angekündigt, was am 20. Mai 2015 online geht: www.youtube.com/watch?v=5NhxB6aQjRY 